\title{
Hipertensos assistidos em serviço de atenção secundária: risco cardiovascular e determinantes sociais de saúde
}

\section{Hypertense individuals assisted in secondary care service: cardiovascular risk and social determinants of health}

André Luiz Cândido Sarmento Drumond Nobre ${ }^{1}$ (D), Cássio de Almeida Lima² (1), Maria José Lages de Oliveira ${ }^{3}$ (D), Davi de Melo Alvarenga Vieira ${ }^{4}$ (D) , Hercílio Martelli Júnior ${ }^{2}$ (D), Simone de Melo Costa ${ }^{3}$ (D)

${ }^{1}$ Centro Estadual de Atenção Especializada (Centro Hiperdia) - Brasília de Minas (MG), Brasil.

${ }^{2}$ Programa de Pós-graduação Stricto Sensu em Ciências da Saúde, Universidade Estadual de Montes Claros (Unimontes) - Montes Claros (MG), Brasil.

${ }^{3}$ Programa de Pós-graduação Stricto Sensu em Cuidado Primário em Saúde, Departamento de Odontologia, Universidade

Estadual de Montes Claros (Unimontes) - Montes Claros (MG), Brasil.

${ }^{4}$ Curso de Graduação em Medicina, Centro de Ensino Superior de Valença - Valença (RJ), Brasil.

Como citar: Nobre ALCSD, Lima CA, Oliveira MJL, Vieira DMA, Martelli Júnior H, Costa SM. Hipertensos assistidos em serviço de atenção secundária: risco cardiovascular e determinantes sociais de saúde. Cad Saúde Colet, 2020;28(3):334-344. https://doi.org/10.1590/1414-462X202028030386

\section{Resumo}

Introdução: Na perspectiva dos determinantes sociais de saúde, a Hipertensão Arterial Sistêmica apresenta complexa natureza multifatorial. É fator de risco cardiovascular, influenciado por aspectos comportamentais, econômicos, sociais, políticos e ambientais. Objetivo: Investigar a relação entre determinantes sociais de saúde e risco cardiovascular global em hipertensos assistidos em serviço da atenção secundária do Sistema Único de Saúde. Método: Estudo seccional analítico, com pacientes do Centro Hiperdia, no norte de Minas Gerais, Brasil. Dados de determinantes sociais foram coletados por meio de questionário estruturado. A classificação do risco cardiovascular (alto, moderado e baixo) deu-se a partir de dados clínicos. A estatística considerou nível de significância 5\%. Resultados: Entre 231 participantes, a maioria (64,1\%) era de alto risco cardiovascular, associado aos dados clínicos: elevada pressão arterial sistólica $(p=0,04)$ e maior tempo de diagnóstico $(p=0,01)$. O alto risco cardiovascular foi associado aos determinantes sociais: satisfação com acesso aos serviços de saúde $(p=0,02)$ e ambiente físico saudável $(p=0,02)$. Conclusão: Apesar de a satisfação com os serviços de saúde e com o ambiente físico terem apresentado resultados reversos, sugere-se fortalecer políticas e práticas de atenção à saúde, que reconheçam diferentes determinantes e previnam a gravidade do risco cardiovascular. Palavras-chave: hipertensão; doença crônica; fatores de risco; vigilância epidemiológica; desigualdades em saúde.

\begin{abstract}
Background: In the perspective of the social determinants of health, Systemic Arterial Hypertension presents complex multifactorial nature. This cardiovascular risk factor is influenced by behavioral, economic, social, political and environmental aspects. Objective: To investigate the relationship between social determinants of health and global cardiovascular risk in hypertensive patients assisted in the secondary care service of the Unified Health System. Method: An analytical sectional study with patients from Centro Hiperdia in Northern Minas Gerais, Brazil. Data of social determinants were collected through a structured questionnaire. The classification of cardiovascular risk (high, moderate and low) was based
\end{abstract}

Trabalho realizado no Centro Estadual de Atenção Especializada (Centro Hiperdia) - Brasília de Minas (MG), Brasil. Correspondência: Cássio de Almeida Lima.E-mail: cassioenf2014@gmail.com

\section{(c) ()}

Este é um artigo publicado em acesso aberto (Open Access) sob a licença Creative Commons Attribution, que permite uso, distribuição e reprodução em qualquer meio, sem restrições desde que o trabalho original seja corretamente citado. 
on clinical data. The statistic considered significance level 5\%. Results: Among 231 participants, the majority (64.1\%) presented high cardiovascular risk, associated with clinical data: high systolic blood pressure $(p=0.04)$ and longer diagnostic time $(p=0.01)$. High cardiovascular risk was associated with social determinants: satisfaction with access to health services $(p=0.02)$ and healthy physical environment $(p=0.02)$. Conclusion: Although satisfaction with the health services and the physical environment presented reversed results, we suggest the strengthening of health care policies and practices that recognize different determinants, and prevent the severity of cardiovascular risk.

Keywords: hypertension; chronic disease; risk factors; epidemiological surveillance; health inequalities.

\section{INTRODUÇÃO}

No cenário brasileiro e internacional, a Hipertensão Arterial Sistêmica (HAS) é considerada um dos grandes desafios para a saúde pública', sendo agravada por sua elevada prevalência e detecção quase sempre tardia ${ }^{2}$. Constitui um dos principais fatores de risco para as doenças cardiovasculares, cerebrovasculares e isquêmicas do coração, assim como para a carga global de doenças em homens e mulheres de todas as idades ${ }^{3}$. Uma revisão sistemática com pesquisas de 90 países revelou prevalência mundial de 31,3\% na população adulta. Estima-se que ocorram 7,1 milhões de mortes anuais decorrentes dessa doença e um crescimento mundial de $60 \%$ dos casos para $2025^{4}$. No Brasil, segundo o estudo "Vigilância de Fatores de Risco e Proteção para Doenças Crônicas por Inquérito Telefônico (VIGITEL) de 2016", a prevalência de HAS é de $25,7 \%$, sendo maior entre mulheres $(27,5 \%)$ do que entre homens $(23,6 \%)^{5}$.

Mesmo sendo conhecidas a eficácia e a efetividade de medidas preventivas e de controle, sejam ou não farmacológicas, a HAS continuará, por décadas, causando impactos para o setor saúde e ônus para a pessoa hipertensa e a sociedade ${ }^{1-3}$. Essa doença pode prejudicar a qualidade de vida do usuário e levar à morte, o que torna prioritárias as ações para evitar tais desfechos ${ }^{6,7}$. Assim, faz-se necessário prestar uma assistência adequada à saúde do hipertenso. Estratificar o risco cardiovascular pode ser uma estratégia efetiva para planejar o cuidado mais qualificado, contribuindo para diminuir as repercussões negativas da evolução da doença $a^{8,9}$.

Para avaliar o risco cardiovascular, foram elaborados e validados instrumentos, entre os quais se destaca o Escore de Risco de Framingham, que corresponde a um percentual de probabilidade de ocorrência de doença cardiovascular nos próximos 10 anos $^{10,11}$. Seguindo os critérios de Framingham, o risco cardiovascular é classificado nas categorias: baixo risco-quando o usuário apresenta uma probabilidade menor que $10 \%$ de eventos cardiovasculares em 10 anos; médio risco - quando há probabilidade entre 10 e 20\%; e, alto risco - corresponde à probabilidade superior a $20 \%{ }^{8,11}$.

É relevante destacar que os fatores relacionados ao risco cardiovascular podem se acumular durante toda a vida e sofrer influência de aspectos comportamentais, econômicos e sociais, e determinantes políticos e ambientais. Acredita-se que os determinantes sociais em saúde possam interferir na exposição de fatores de risco cardiovascular ${ }^{12}$. Assim, a manifestação da HAS não pode ser explicada apenas por relação de causa e efeito, mas pelo contexto social e pelo estilo de vida em que o indivíduo se encontra inserido ${ }^{13}$.

Na perspectiva de determinantes sociais do processo saúde-doença, estes são definidos como as condições sociais em que as pessoas crescem, vivem, trabalham e envelhecem. São os fatores e mecanismos pelos quais as condições sociais afetam a saúde, que potencialmente podem ser alterados pelas ações baseadas em informação e, principalmente, pelas políticas públicas nas esferas social e da saúde ${ }^{14-17}$. Destaca-se o modelo de Dahlgren e Whitehead, adotado pela Comissão sobre Determinantes Sociais de Saúde (CDSS) da Organização Mundial da Saúde (OMS). Ele incorpora diferentes determinantes: características individuais; comportamento e estilos de vida; condições de vida e de trabalho; disponibilidade de alimentos e acesso a ambientes saudáveis e serviços essenciais, e condições socioeconômicas, culturais e ambientais da sociedade ${ }^{12}$.

Logo, é indubitável a relevância de pesquisas que evidenciem os fatores relacionados à HAS e ao risco cardiovascular, de acordo com um conjunto de características individuais, socioculturais e econômicas ${ }^{18,19}$, que devem ser contextualizadas em uma concepção mais ampliada do processo saúde-doença-cuidado. E o conhecimento sobre os determinantes 
sociais pode auxiliar gestores e profissionais da saúde na efetivação de políticas públicas e práticas em saúde coerentes com a realidade ${ }^{20,21}$ - especialmente na região norte de Minas Gerais (MG), Brasil, onde existem lacunas de evidências sobre o assunto. São necessários estudos envolvendo um grupo populacional específico inserido em um cenário particular, que identifiquem determinantes sociais a serem considerados em estratégias de prevenção e controle da $\mathrm{HAS}^{22}$ mais apropriadas aos estratos de Framingham.

Com base no exposto, este trabalho teve como hipótese que o alto risco cardiovascular se associa aos piores determinantes sociais. Objetivou-se investigar a relação entre determinantes sociais de saúde e risco cardiovascular global em hipertensos assistidos em serviço da atenção secundária do Sistema Único de Saúde (SUS).

\section{MÉTODO}

Trata-se de estudo seccional analítico, que contou com a participação de hipertensos, assistidos no Centro Estadual de Atenção Especializada (Centro Hiperdia), de um município do norte do estado de Minas Gerais, Brasil.

O Centro Hiperdia, em nível da Atenção Secundária à Saúde, constitui a principal estratégia do Governo de Minas Gerais para o enfrentamento das condições crônicas de saúde: Hipertensão Arterial, Doenças Cardiovasculares, Diabetes Mellitus e Doença Renal Crônica. O serviço é referência na atenção secundária para hipertensos de alto risco cardiovascular, de acordo com estratificação pelos Critérios de Framingham. Os usuários são encaminhados via serviço de Atenção Primária à Saúde (APS), após avaliação médica ${ }^{23}$.

O tamanho da amostra foi definido por cálculo amostral, a partir dos 710 cadastros de adultos e idosos. Considerou-se o erro amostral de 5\%, intervalo de confiança $95 \%$ e prevalência do desfecho de $50 \%$, por possibilitar a obtenção de um " $n$ " maior. O número amostral foi definido em 250 participantes, sem acréscimo de compensação para possíveis não respostas e perdas.

Para esta investigação, foram determinados os seguintes critérios de inclusão: adultos e idosos assistidos no Centro Hiperdia por terem sido diagnosticados com hipertensão arterial, por médico, a partir de métodos objetivos de medida da pressão arterial, e que concordassem em participar do estudo mediante a leitura e assinatura do Termo de Consentimento Livre e Esclarecido (TCLE). Como critério de exclusão, adotou-se: paciente sem condições de responder ao questionário, seja por questões físicas, cognitivas ou outras deficiências, como auditivas, conforme informação do acompanhante.

A coleta de dados deu-se no segundo semestre de 2013. Os dados foram coletados por meio de um questionário estruturado, desenvolvido pelos pesquisadores, a partir dos fatores de risco cardiovascular e conforme a teoria dos determinantes sociais de saúde, segundo modelo de Dahlgren e Whitehead ${ }^{12}$. A coleta se deu no formato de entrevista conduzida em ambiente reservado para esse fim, anteriormente à consulta cardiológica, consecutivamente, até atingir os 250 participantes. Não houve recusa de participação. Após aplicação do questionário, as informações clínicas foram complementadas a partir dos registros nos prontuários de saúde de cada paciente: índice de massa corporal (IMC), classificado em normal, sobrepeso e obeso, a partir de medidas objetivas de peso e estatura, que são registradas nos prontuários dos pacientes na consulta médica; tempo de diagnóstico da HAS; medidas da pressão arterial diastólica e sistólica registradas na última consulta médica; história familiar de hipertensão, e classificação do risco cardiovascular pelos Critérios de Framingham ${ }^{11}$. Todos os dados foram coletados por um único pesquisador, médico do Centro Hiperdia.

As variáveis relativas aos determinantes foram:

- Características individuais/fatores hereditários: sexo (feminino, masculino); cor autorreferida (branca, preta, amarela, parda e indígena); idade na data da entrevista e faixa etária (adulta de 18 a 59 anos e idosa de 60 ou mais anos); IMC (normal, sobrepeso/obeso); história familiar de hipertensão (sim, não);

- Comportamento e estilos de vida: restrição no uso do sal (sim, não), no uso de gordura (sim, não); exercício físico (sim - para toda atividade planejada e repetitiva para melhorar e manter a aptidão física, como caminhada, esporte; não); uso de álcool e tabaco (nunca 
e ex/atual para qualquer quantidade de consumo de bebida alcoólica ou de tabaco); oportunidade de lazer (não/muito pouco/mais ou menos, bastante/extremamente);

- Redes sociais e comunitárias: disponibilidade de informações (não/mais ou menos, bastante/extremamente); satisfação com relações pessoais (sim, não);

- $\quad$ Condições de vida e de trabalho: escolaridade (analfabeto, alfabetizado); renda familiar mensal em reais e categorizada (até um salário mínimo e mais de um salário mínimo); tempo de diagnóstico da HAS (diferença entre o tempo do registro no prontuário até a data da coleta de dados da pesquisa - variável que tem relação com serviços de saúde e, por isso, não foi considerada como característica individual); satisfação com acesso aos serviços de saúde (insatisfeito, satisfeito);

- Condições socioeconômicas, culturais e ambientais: ambiente físico saudável (não/pouco/mais ou menos, bastante/extremamente); satisfação com local de moradia (insatisfeito/mais ou menos, satisfeito) e com meios de transporte (insatisfeito/mais ou menos, satisfeito).

Previamente ao estudo principal, com o propósito de testar a clareza das questões do questionário, realizou-se um estudo-piloto com 10 usuários do Centro Hiperdia. Os dados do piloto foram considerados no estudo principal.

O banco de dados foi alimentado pelo mesmo pesquisador que efetuou a coleta de dados, preservando o anonimato das informações. Os dados foram conferidos por um segundo pesquisador, a partir dos registros contidos nos questionários da pesquisa.

A análise estatística foi realizada empregando-se o programa IBM Statistical Package for the Social Sciences (SPSS) Statistics, versão $22.0^{\oplus}$. O teste Anova foi utilizado para comparar as médias da pressão arterial sistólica e do tempo de diagnóstico da HAS entre os três grupos de risco cardiovascular: alto, moderado e baixo. As associações entre risco cardiovascular e determinantes sociais de saúde foram mensuradas por meio do teste Qui-quadrado de Pearson $\left(x^{2}\right)$. Considerou-se um nível de significância estatística de $5 \%(p<0,05)$.

$\mathrm{O}$ projeto de pesquisa que deu origem a este trabalho foi aprovado pelo Comitê de Ética em Pesquisa da Universidade Estadual de Montes Claros (Parecer Consubstanciado n. ${ }^{\circ} 226.732 / 2013$ ).

\section{RESULTADOS}

Entre os 250 indivíduos que responderam ao questionário, houve perda de informações nos prontuários de saúde de 19 participantes (7,6\%). Portanto, o atual estudo contou com a participação de 231 hipertensos, com informações completas e necessárias aos propósitos da investigação. Verificou-se maior frequência de participantes do sexo feminino (71,0\%). A idade variou de 23 a 95 anos, com média de 60( $\pm 13,37)$ anos. A faixa etária correspondente aos adultos (18 a 59 anos) apresentou um percentual (51,5\%) pouco maior que os idosos. Em relação à cor de pele autodeclarada, grande parte dos pesquisados informou ser parda $(68,9 \%)$, seguida de branca $(17,7 \%)$, preta $(11,3 \%)$, amarela $(1,3 \%)$, indígena $(0,4 \%)$ e $1,3 \%$ não respondeu a essa questão.

Conforme o risco cardiovascular, $13,0 \%$ dos hipertensos foram classificados em baixo risco, $22,9 \%$ em risco moderado e $64,1 \%$ em alto risco. A média da pressão sistólica no grupo de alto risco foi $140,27( \pm 24,47) \mathrm{mmHg}$, sendo constatada diferença significante entre os grupos $(p=0,04)$. A maior média do tempo de diagnóstico da doença também foi para o grupo de hipertensos de alto risco cardiovascular $(p=0,01)$ (Tabela 1).

Na Tabela 2, as variáveis que apresentaram associações estatisticamente significantes com o alto risco cardiovascular foram: satisfação com o acesso aos serviços de saúde $(p=0,02)$ e considerar saudável o ambiente físico onde reside $(p=0,02)$.

\section{DISCUSSÃO}

Este estudo investigou a relação entre os determinantes sociais de saúde e o risco cardiovascular global entre hipertensos assistidos em um centro de referência. As variáveis relacionadas ao risco cardiovascular foram: maior pressão arterial sistólica; maior tempo de 
Tabela 1. Comparação entre médias da pressão arterial sistólica e do tempo de diagnóstico da Hipertensão Arterial Sistêmica (HAS), e classificação de risco. Centro Hiperdia, Minas Gerais, Brasil (2013)

\begin{tabular}{|c|c|c|c|c|c|c|c|c|}
\hline \multirow{2}{*}{$\begin{array}{c}\text { Classificação de Risco } \\
\text { Cardiovascular de } \\
\text { Framingham (em } 10 \text { anos) }\end{array}$} & \multirow{2}{*}{$\mathbf{n}$} & \multirow{2}{*}{$\%$} & \multicolumn{3}{|c|}{$\begin{array}{l}\text { Pressão Arterial Sistólica } \\
(\mathrm{mmHg})\end{array}$} & \multicolumn{3}{|c|}{$\begin{array}{c}\text { Tempo de diagnóstico } \\
\text { da HAS (em anos) }\end{array}$} \\
\hline & & & Média & DP* & $\begin{array}{l}\text { Valor } \\
\text { de } p\end{array}$ & Média & DP* & $\begin{array}{l}\text { Valo } \\
\text { de } p\end{array}$ \\
\hline Baixo $(<10 \%)$ & 30 & 13,0 & 130,50 & 22,45 & \multirow[t]{4}{*}{0,04} & 8,34 & 6,92 & \multirow[t]{4}{*}{0,01} \\
\hline Moderado (10 a 20\%) & 53 & 22,9 & 133,58 & 16,18 & & 11,09 & 8,51 & \\
\hline Alto $(>20 \%)$ & 148 & 64,1 & 140,27 & 24,47 & & 13,66 & 9,63 & \\
\hline Total & 231 & 100,0 & - & - & & - & - & \\
\hline
\end{tabular}

*DP: desvio padrão da média

Tabela 2. Determinantes Sociais de Saúde conforme classificação de risco cardiovascular de Framingham. Centro Hiperdia, Minas Gerais, Brasil (2013)

\begin{tabular}{|c|c|c|c|c|c|c|c|}
\hline \multirow{4}{*}{$\begin{array}{l}\text { Determinantes sociais } \\
\text { de saúde de Dahlgren } \\
\text { e Whitehead (1991) }\end{array}$} & \multicolumn{7}{|c|}{ Classificação de Risco Cardiovascular de Framingham (em 10 anos) } \\
\hline & \multirow{2}{*}{\multicolumn{2}{|c|}{$\begin{array}{c}\text { Baixo } \\
(<10 \%)\end{array}$}} & \multirow{2}{*}{\multicolumn{2}{|c|}{$\begin{array}{l}\text { Moderado } \\
(10 \text { a } 20 \%)\end{array}$}} & \multirow{2}{*}{\multicolumn{2}{|c|}{$\begin{array}{c}\text { Alto } \\
(>20 \%)\end{array}$}} & \multirow{3}{*}{$\begin{array}{l}\text { Valor } \\
\text { de } p\end{array}$} \\
\hline & & & & & & & \\
\hline & $\mathbf{n}$ & $\%$ & $\mathbf{n}$ & $\%$ & $\mathbf{n}$ & $\%$ & \\
\hline \multicolumn{8}{|l|}{ Sexo } \\
\hline Feminino & 23 & 14,0 & 38 & 23,2 & 103 & 62,8 & 0,73 \\
\hline Masculino & 7 & 10,4 & 15 & 22,4 & 45 & 67,2 & \\
\hline \multicolumn{8}{|l|}{ Cor autodeclarada* } \\
\hline Branca & 10 & 24,4 & 6 & 14,6 & 25 & 61,0 & \\
\hline Preta & 4 & 15,4 & 3 & 11,5 & 19 & 73,1 & 0,13 \\
\hline Amarela & 1 & 33,3 & 1 & 33,3 & 1 & 33,3 & \\
\hline Parda & 14 & 8,9 & 42 & 26,8 & 101 & 64,3 & \\
\hline Indígena & 0 & 0,0 & 0 & 0,0 & 1 & 100,0 & \\
\hline \multicolumn{8}{|l|}{ Faixa etária } \\
\hline Adulto (18-59 anos) & 18 & 15,1 & 29 & 24,4 & 72 & 60,5 & 0,46 \\
\hline Idoso ( $\geq 60$ anos) & 12 & 10,7 & 24 & 21,4 & 76 & 67,9 & \\
\hline \multicolumn{8}{|l|}{$\begin{array}{l}\text { Classificação do Índice } \\
\text { de Massa Corporal }\end{array}$} \\
\hline Normal & 12 & 14,5 & 21 & 25,3 & 50 & 60,2 & 0,66 \\
\hline Sobrepeso/Obeso & 18 & 12,2 & 32 & 21,6 & 98 & 66,2 & \\
\hline \multicolumn{8}{|l|}{$\begin{array}{l}\text { História familiar de } \\
\text { hipertensão }\end{array}$} \\
\hline Sim & 26 & 12,9 & 45 & 22,2 & 131 & 64,9 & 0,79 \\
\hline Não & 4 & 13,8 & 8 & 27,6 & 17 & 58,6 & \\
\hline \multicolumn{8}{|l|}{ Restrição ao uso do sal } \\
\hline Sim & 29 & 13,4 & 52 & 23,9 & 136 & 62,7 & 0,21 \\
\hline Não & 1 & 7,1 & 1 & 7,1 & 12 & 85,7 & \\
\hline
\end{tabular}

Ausência de respostas: * 3 ; **1 
Tabela 2. Continuação...

\begin{tabular}{|c|c|c|c|c|c|c|c|}
\hline \multirow{4}{*}{$\begin{array}{l}\text { Determinantes sociais } \\
\text { de saúde de Dahlgren } \\
\text { e Whitehead (1991) }\end{array}$} & \multicolumn{7}{|c|}{ Classificação de Risco Cardiovascular de Framingham (em 10 anos) } \\
\hline & \multirow{2}{*}{\multicolumn{2}{|c|}{$\begin{array}{c}\text { Baixo } \\
(<10 \%)\end{array}$}} & \multirow{2}{*}{\multicolumn{2}{|c|}{$\begin{array}{l}\text { Moderado } \\
\text { (10 a 20\%) }\end{array}$}} & \multirow{2}{*}{\multicolumn{2}{|c|}{$\begin{array}{c}\text { Alto } \\
(>20 \%)\end{array}$}} & \multirow{3}{*}{$\begin{array}{l}\text { Valor } \\
\text { de p }\end{array}$} \\
\hline & & & & & & & \\
\hline & $\mathbf{n}$ & $\%$ & $\mathbf{n}$ & $\%$ & $\mathbf{n}$ & $\%$ & \\
\hline \multicolumn{8}{|l|}{$\begin{array}{l}\text { Restrição ao uso da } \\
\text { gordura }\end{array}$} \\
\hline Sim & 26 & 12,5 & 46 & 22,1 & 136 & 65,4 & 0,46 \\
\hline Não & 4 & 17,4 & 7 & 30,4 & 12 & 52,2 & \\
\hline \multicolumn{8}{|l|}{ Exercício físico } \\
\hline Sim & 9 & 10,1 & 21 & 23,6 & 59 & 66,3 & 0,59 \\
\hline Não & 21 & 14,8 & 32 & 22,5 & 89 & 62,7 & \\
\hline \multicolumn{8}{|l|}{ Etilismo** } \\
\hline Nunca & 19 & 11,1 & 39 & 22,8 & 113 & 66,1 & 0,33 \\
\hline Ex-etilista/atual & 11 & 18,6 & 13 & 22,0 & 35 & 59,3 & \\
\hline \multicolumn{8}{|l|}{ Tabagismo } \\
\hline Nunca & 20 & 11,3 & 44 & 24,7 & 114 & 64,0 & 0,24 \\
\hline Ex-tabagista /atual & 10 & 18,9 & 9 & 17,0 & 34 & 64,1 & \\
\hline \multicolumn{8}{|l|}{$\begin{array}{l}\text { Oportunidade de } \\
\text { atividades de lazer }\end{array}$} \\
\hline $\begin{array}{l}\text { Não/muito pouco/mais } \\
\text { ou menos }\end{array}$ & 26 & 13,4 & 43 & 22,2 & 125 & 64,4 & \\
\hline Bastante/extremamente & 4 & 10,8 & 10 & 27,0 & 24 & 58,5 & 0,78 \\
\hline \multicolumn{8}{|l|}{$\begin{array}{l}\text { Informações } \\
\text { disponíveis }\end{array}$} \\
\hline Não/mais ou menos & 20 & 14,6 & 29 & 21,2 & 88 & 64,2 & 0,57 \\
\hline Bastante/extremamente & 10 & 10,6 & 24 & 25,6 & 60 & 63,8 & \\
\hline \multicolumn{8}{|l|}{$\begin{array}{l}\text { Satisfação com } \\
\text { relações pessoais }\end{array}$} \\
\hline Não & 5 & 15,6 & 7 & 21,9 & 20 & 62,5 & 0,89 \\
\hline Sim & 25 & 12,6 & 46 & 23,1 & 128 & 64,3 & \\
\hline \multicolumn{8}{|l|}{ Escolaridade } \\
\hline Analfabeto & 10 & 14,3 & 14 & 20,0 & 46 & 65,7 & 0,76 \\
\hline Alfabetizado & 20 & 12,4 & 39 & 24,2 & 102 & 63,4 & \\
\hline \multicolumn{8}{|l|}{ Renda familiar mensal * } \\
\hline Até 1 salário mínimo & 14 & 13,5 & 27 & 26,0 & 63 & 60,5 & 0,55 \\
\hline $\begin{array}{l}\text { Mais de } 1 \text { salário } \\
\text { mínimo }\end{array}$ & 16 & 12,9 & 25 & 20,2 & 83 & 66,9 & \\
\hline
\end{tabular}

Ausência de respostas: *3; **1 
Tabela 2. Continuação...

\begin{tabular}{|c|c|c|c|c|c|c|c|}
\hline \multirow{4}{*}{$\begin{array}{l}\text { Determinantes sociais } \\
\text { de saúde de Dahlgren } \\
\text { e Whitehead (1991) }\end{array}$} & \multicolumn{7}{|c|}{ Classificação de Risco Cardiovascular de Framingham (em 10 anos) } \\
\hline & \multirow{2}{*}{\multicolumn{2}{|c|}{$\begin{array}{c}\text { Baixo } \\
(<10 \%)\end{array}$}} & \multirow{2}{*}{\multicolumn{2}{|c|}{$\begin{array}{l}\text { Moderado } \\
\text { (10 a } 20 \%)\end{array}$}} & \multirow{2}{*}{\multicolumn{2}{|c|}{$\frac{\text { Alto }}{(>20 \%)}$}} & \multirow{3}{*}{$\begin{array}{l}\text { Valor } \\
\text { de } p\end{array}$} \\
\hline & & & & & & & \\
\hline & $\mathbf{n}$ & $\%$ & $\mathbf{n}$ & $\%$ & $\mathbf{n}$ & $\%$ & \\
\hline \multicolumn{8}{|l|}{$\begin{array}{l}\text { Satisfação com acesso } \\
\text { aos serviços de saúde }\end{array}$} \\
\hline Insatisfeito & 18 & 21,4 & 18 & 21,4 & 48 & 57,2 & 0,02 \\
\hline Satisfeito & 12 & 8,2 & 35 & 23,8 & 100 & 68,0 & \\
\hline \multicolumn{8}{|l|}{$\begin{array}{l}\text { Considera saudável o } \\
\text { ambiente físico** }\end{array}$} \\
\hline $\begin{array}{l}\text { Não/pouco/mais ou } \\
\text { menos }\end{array}$ & 19 & 20,2 & 18 & 19,1 & 57 & 60,6 & 0,02 \\
\hline Bastante/extremamente & 11 & 8,1 & 34 & 25,0 & 91 & 66,9 & \\
\hline \multicolumn{8}{|l|}{$\begin{array}{l}\text { Satisfação com o local } \\
\text { de moradia }\end{array}$} \\
\hline $\begin{array}{l}\text { Insatisfeito/mais ou } \\
\text { menos }\end{array}$ & 10 & 19,6 & 9 & 17,7 & 32 & 62,7 & 0,22 \\
\hline Satisfeito & 20 & 11,1 & 44 & 24,4 & 116 & 64,4 & \\
\hline \multicolumn{8}{|l|}{$\begin{array}{l}\text { Satisfação com os } \\
\text { meios de transporte }\end{array}$} \\
\hline $\begin{array}{l}\text { Insatisfeito/mais ou } \\
\text { menos }\end{array}$ & 18 & 17,1 & 26 & 24,8 & 61 & 58,1 & 0,55 \\
\hline Satisfeito & 12 & 9,5 & 27 & 21,4 & 87 & 69,0 & \\
\hline
\end{tabular}

Ausência de respostas: * $3 ;{ }^{* * 1}$

diagnóstico da doença; satisfação com o acesso aos serviços de saúde, e percepção de ser saudável o ambiente físico de residência. Essas variáveis integram os determinantes sociais, na teoria de Dalhgren e Whitehead ${ }^{12}$. Variáveis que tratam de comportamento e estilos de vida, e das redes sociais e comunitárias não ficaram associadas à gravidade do risco cardiovascular. Os resultados reforçam a necessidade de discussão da natureza multifatorial complexa que envolve as Doenças Crônicas Não Transmissíveis (DCNTs) e os determinantes sociais de saúde.

A maioria dos participantes foi classificada como de alto risco cardiovascular. Esse resultado era esperado, uma vez que o estudo foi conduzido com pessoas referenciadas ao setor de atenção secundária para tratamento da HAS de alto risco. Entretanto, entre os participantes, 35,9\% apresentaram moderado/baixo risco cardiovascular.

As pessoas classificadas como de alto risco cardiovascular pelos critérios de Framingham requerem cuidados especiais, considerando o maior risco de complicações e de mortalidade. As ações propostas pela equipe multiprofissional do Centro Hiperdia possibilitam monitorar e efetuar intervenções mais efetivas na dimensão preventiva do serviço ${ }^{24}$. Esse serviço é referência no atendimento secundário para os hipertensos de alto risco cardiovascular. Todavia, como observado nesta investigação, por falhas no referenciamento dos casos, chegam a esse setor pessoas classificadas em baixo e moderado risco. Logo, para que a rede de atenção à saúde funcione de forma resolutiva, os referenciamentos de um nível para outro devem respeitar critérios e parâmetros definidos pelo sistema de governança. Na discussão sobre as DCNTs e o sistema público de saúde, a questão da escassez de recursos está sempre em pauta. Essa insuficiência é, de fato, notória e requer equidade na distribuição dos recursos, implicando no 
correto referenciamento entre os diferentes níveis de atenção, a fim de garantir a integralidade da assistência ${ }^{23,24}$.

O maior valor da pressão arterial correspondeu à maior gravidade de risco cardiovascular, resultado compatível com critérios biológicos. E a maior média do tempo de diagnóstico de HAS foi para o alto risco cardiovascular. Esse resultado sugere que o maior tempo de diagnóstico não colaborou para o monitoramento adequado da HAS, no intuito de controlá-la e prevenir o agravamento do risco cardiovascular. Nesse sentido, questiona-se: onde se encontra a fragilidade? No indivíduo, que não aderiu à terapia proposta e não adotou as medidas de autocuidado, ou nos profissionais de saúde, que não manejaram, adequadamente, a condição crônica, por meio de medidas preventivas e de promoção da saúde, além da prescrição medicamentosa? Ou a fragilidade está no modelo assistencial da saúde pública, que ainda privilegia atenção às condições agudas, em detrimento das condições crônicas?

Dessa maneira, observa-se que o maior tempo de convivência com a doença contribui para o agravamento da HAS, situação propícia ao aparecimento de complicações e agravos, como as lesões secundárias: problemas renais e $\operatorname{cardíacos}^{13,24}$. Isso repercute em ônus à sociedade, devido ao aumento de doentes crônicos e à perda da força de trabalho, com afastamentos e aposentadorias motivadas pela incapacidade gerada pela doença, além dos gastos com serviços de saúde e medicação, entre outros ${ }^{13}$. Trata-se de uma realidade preocupante que evidencia a necessidade de um melhor planejamento estratégico das intervenções assistenciais, para promover mudanças no estilo de vida de grupos de risco ${ }^{6}$.

Assim, não se trata apenas de ofertar uma atenção em termos quantitativos, com mais profissionais e serviços de referência, mas também qualitativos, ou seja, relativos à qualidade do cuidado. É necessária uma abordagem integral, de modo a otimizar a adesão ao tratamento e ao controle da pressão arterial, com controles pressóricos mais acurados, acompanhamento criterioso e revisão de medidas preventivas e terapêuticas adotadas. Também é preciso focar os fatores de risco e a associação destes nas pessoas com HAS, com consequente melhora na qualidade de vida e redução da morbimortalidade ${ }^{7,25}$. Nesse contexto, destaca-se a relação profissional-usuário, que requer o diálogo efetivo para favorecer a compreensão do tratamento; o uso adequado dos medicamentos, e a aceitação dos limites impostos pela dieta hipossódica, a qual, muitas vezes, pode se contrapor aos costumes da comunidade ${ }^{7}$.

No que tange aos comportamentos e estilos de vida, nenhuma das variáveis se manteve associada à classificação de risco cardiovascular, apesar de a literatura informar que fatores, como maior uso de sal e gordura, falta de atividade física, uso de álcool e tabaco, e carência de oportunidades de lazer, contribuem para o agravamento da $\mathrm{HAS}^{3,7,8,22,26}$.

As variáveis de redes sociais e comunitárias, que envolvem a disponibilidade de informações e as relações pessoais, não tiveram relação com a classificação de risco cardiovascular. Contudo, é importante ressaltar que o nível de coesão social é de fundamental importância para a saúde da população ${ }^{14}$. O menor capital social torna-se um mecanismo para as iniquidades socioeconômicas, por influenciar negativamente na saúde. Países com frágil coesão social, devido às iniquidades, pouco investem em capital humano e nas redes de apoio social. Além do mais, nesses países, encontra-se uma menor participação na definição de políticas públicas. Por outro lado, não são as sociedades mais ricas que possuem os melhores níveis de saúde, mas sim aquelas mais igualitárias e com alta coesão social ${ }^{27}$.

No Brasil, a Comissão Nacional sobre os Determinantes Sociais da Saúde (CNDSS) recomenda a participação social para fortalecer os mecanismos de gestão participativa ${ }^{28}$. Para tanto, é importante adotar ações que disseminem as informações e capacitem os líderes comunitários acerca dos determinantes sociais da saúde, além de estratégias para o fortalecimento da comunicação social. Assim, confere-se a base de apoio político às ações sobre os determinantes e o empoderamento da população vulnerável, para participar das decisões sobre a saúde e o bem-estar ${ }^{17,21,28}$.

O maior percentual de pessoas satisfeitas com o acesso aos serviços de saúde era de alto risco cardiovascular. Achado interessante nesta investigação, já que se esperava ser, a insatisfação, a categoria associada à maior gravidade do risco cardiovascular. Acredita-se que este resultado esteja relacionado ao apoio recebido no nível de atenção secundária, por 
envolver uma atenção diferenciada, com equipe multiprofissional e em melhor estrutura física que as unidades da APS, aspectos estes que podem ter influenciado, positivamente, na opinião dos pesquisados.

É válido salientar que, nos casos de pessoas com alto risco cardiovascular, o investimento público se justifica, na medida em que as ações na atenção secundária - a exemplo das executadas no Centro Hiperdia - oferecem melhor custo-benefício e estão ancoradas no modelo assistencial direcionado à atenção às condições crônicas ${ }^{24}$. Ademais, a região Sudeste, onde se localiza o cenário desta investigação, assim como a região Sul, quando comparadas às demais regiões brasileiras, possuem melhor estrutura de serviços de saúde, tanto do setor público quanto do setor privado, para acesso ao diagnóstico médico e ao tratamento da $\mathrm{HAS}^{29}$, sendo essa outra hipótese para o achado deste trabalho.

O acesso garantido aos serviços de saúde pública se faz essencial para o usuário hipertenso. Especialmente aquele em situação de alto risco cardiovascular deve ser assistido na APS pelas equipes de saúde da família, visando à adesão ao tratamento; ao autocuidado, e à responsabilidade compartilhada. Entretanto, embora a Estratégia de Saúde da Família seja cenário privilegiado para intervenções de base comunitária direcionadas à prevenção integrada dos fatores de risco cardiovascular, há fragilidades no manejo do portador de HAS ${ }^{30}$.

Nos determinantes sociais de saúde que tratam das condições socioeconômicas, culturais e ambientais, a percepção do ambiente físico saudável esteve associada a um maior percentual de pessoas com alto risco cardiovascular. Resultado reverso ao esperado, uma vez que, segundo a teoria adotada, as situações insatisfatórias é que determinariam a pior condição de saúde ${ }^{12}$. Entre os elementos que contribuem para a multideterminação da HAS, encontra-se o ambiente físico ${ }^{3}$. Tal elemento remete à promoção da saúde, ampliando a atenção à saúde do hipertenso para ambientes e cidades saudáveis. Se a perspectiva que vincula saúde e condições de vida é trazida à tona, múltiplos determinantes, tais como o ambiente onde o indivíduo se encontra, emergem como relacionados a uma vida saudável'.

Estudos realçam que a relação entre ambientes saudáveis e a saúde cardiovascular pode ser mediada pela prática de atividades físicas, oportunidades de lazer e pela melhoria das condições psicossociais, como redução do estresse e fomento do contato e da coesão social ${ }^{31,32}$. Tais ambientes se caracterizam como fatores contextuais na saúde da população, os quais são pertinentes na orientação de medidas de promoção da saúde, norteadas ao controle das diversas DCNTs ${ }^{32}$. É válido salientar que o "Plano de Ações Estratégicas para o Enfrentamento das Doenças Crônicas Não Transmissíveis no Brasil 2011-2022"26 propõe ações de abrangência populacional intersetoriais, que extrapolam o setor saúde. As ações relacionadas à promoção da saúde e à prevenção de agravos devem estar inseridas nas prioridades do setor saúde, em todos os seus níveis de gestão e atenção ${ }^{22,26}$.

Por fim, os resultados do atual trabalho devem ser considerados à luz de certas limitações. Avaliou-se a relação entre os determinantes sociais de saúde com a gravidade do risco cardiovascular, mas, por se tratar de estudo transversal, não é possível afirmar causalidade das associações. Apesar de a amostra ter sido definida a partir de cálculo amostral para representar o total de cadastrados, o estudo foi realizado com parte dos usuários do serviço e houve uma pequena perda $(7,6 \%)$ do previsto. O levantamento dos dados por meio de um questionário pode ter acarretado um possível viés de informação e de memória. Para amenizar essa limitação, a entrevista foi conduzida em ambiente reservado e com cada participante, por vez. O fato de os dados terem sido coletados em um centro especializado para tratamento de HAS de alto risco configura outra limitação, uma vez que a maioria dos pacientes já apresentará risco cardiovascular elevado e era previsto uma maior participação de pacientes classificados em alto risco cardiovascular. No entanto, a classificação de risco cardiovascular não foi critério de inclusão no estudo, o que contribuiu para a participação de quase $36 \%$ dos participantes com riscos moderado/baixo.

O estudo evidenciou que a maioria dos determinantes sociais não apresentou associação estatisticamente significante com o maior risco cardiovascular, corroborando a complexa natureza multifatorial da HAS. A elevada pressão arterial sistólica e o maior tempo de diagnóstico foram observados no estrato de alto risco cardiovascular. Os hipertensos que 
manifestaram satisfação com os serviços de saúde e com o ambiente físico em que residem apresentaram relação com alto risco cardiovascular, resultados estes reversos ao esperado.

Esses achados contrariam a hipótese desta pesquisa, em parte, pois nem todos os piores determinantes sociais de saúde se relacionaram com a maior gravidade do risco cardiovascular, exigindo cautela na análise teórica das DCNTs.

A HAS é um problema de saúde pública, com alto risco de eventos cardiovasculares, o que torna necessário aprimorar cada vez mais a qualidade da assistência aos hipertensos, sobretudo na APS e nos serviços de referência. Também é preciso buscar uma abordagem mais integral, no sentido de otimizar a adesão ao tratamento e realizar um acompanhamento criterioso. Logo, sugere-se o fortalecimento de políticas públicas com foco nos modos de viver, na efetivação de práticas de promoção da saúde, bem como nas ações intersetoriais e integradas, que reconheçam os diferentes determinantes de saúde.

\section{REFERÊNCIAS}

1. Santos ZMSA. Hipertensão arterial - um problema de saúde pública. Rev Bras Promoç Saúde. 2011;24(4):285-6. http://dx.doi.org/10.5020/18061230.2011.p285.

2. Ferreira RA, Barreto SM, Giatti L. Hipertensão arterial referida e utilização de medicamentos de uso contínuo no Brasil: um estudo de base populacional. Cad Saude Publica. 2014;30(4):815-26. http://dx.doi. org/10.1590/0102-311X00160512. PMid:24896056.

3. Malta DC, Bernal RTI, Andrade SSCA, Silva MMA, Velasquez-Melendez G. Prevalence of and factors associated with self-reported high blood pressure in Brazilian adults. Rev Saude Publica. 2017;51(Suppl 1):11s. http://dx.doi.org/10.1590/s1518-8787.2017051000006. PMid:28591346.

4. Mills KT, Bundy JD, Kelly TN, Reed JE, Kearney PM, Reynolds K, et al. Global disparities of hypertension prevalence and control clinical perspective. Circulation. 2016;134(6):441-50. http://dx.doi.org/10.1161/ CIRCULATIONAHA.115.018912. PMid:27502908.

5. Brasil. Ministério da Saúde. Secretaria de Vigilância em Saúde. Departamento de Vigilância de Doenças e Agravos Não Transmissíveis e Promoção da Saúde. Vigitel Brasil 2016: vigilância de fatores de risco e proteção para doenças crônicas por inquérito telefônico: estimativa sobre frequência e distribuição sociodemográfica de fatores de risco e proteção para doenças crônicas nas capitais dos 26 estados brasileiros e no Distrito Federal em 2016. Brasília: Ministério da Saúde; 2017. 160 p.

6. Nascimento ES, Castelo Branco MPF, Moreira AKF, Hazime FA. Estratificação do risco cardiovascular global em hipertensos atendidos numa unidade de saúde da família de Parnaíba, Piauí. Rev Bras Promoç Saúde. 2012;25(3):287-94. http://dx.doi.org/10.5020/18061230.2012.p287.

7. Tavares RS, Silva DMGV, Dal Sasso GTM, Padilha MICS, Santos CRM. Cardiovascular risk factors: the study included hypertensive people in a popular neighborhood in the amazon region. Cienc Cuid Saude. 2014;13(1):4-11.

8. Brasil. Ministério da Saúde. Secretaria de Atenção à Saúde. Departamento de Atenção Básica. Estratégias para o cuidado da pessoa com doença crônica: hipertensão arterial sistêmica. Brasília (DF): Ministério da Saúde; 2013.

9. Paula EA, Paula RB, Costa DMN, Colugnati FAB, Paiva EP. Cardiovascular risk assessment in hypertensive patients. Rev Lat Am Enfermagem. 2013;21(3):820-7. http://dx.doi.org/10.1590/S010411692013000300023. PMid:23918030.

10. Wilson PWF, D'Agostino RB, Levy D, Belanger AM, Silbershatz H, Kannel WB. Prediction of coronary heart disease using risk factor categories. Circulation. 1998;97(18):1837-47. http://dx.doi.org/10.1161/01. CIR.97.18.1837. PMid:9603539.

11. D'Agostino RB Sr, Vasan RS, Pencina MJ, Wolf PA, Cobain M, Massaro JM, et al. General cardiovascular risk profile for use in primary care: the Framingham heart study. Circulation. 2008;117(6):743-53. http://dx.doi. org/10.1161/CIRCULATIONAHA.107.699579. PMid:18212285.

12. Dahlgren G, Whitehead M. Policies and strategies to promote social equity in health. Stockholm: Institute for Future Studies; 1991.

13. Salcedo-Barrientos DM, Siqueira EFG, Egry EY. Determinantes sociais e hipertensão arterial: um desafio na saúde coletiva. Avances Enfermería. 2013;31(1):72-86.

14. Commission on Social Determinants of Health. Closing the gap in a generation: health equity through action on the social determinants of health. Final report of the Commission on Social Determinants of Health. Geneva: World Health Organization; 2008. 
15. Buss PM, Pellegrini Filho A. A saúde e seus determinantes sociais. Phisis Rev Saude Coletiva. 2007;17(1):7793. http://dx.doi.org/10.1590/S0103-73312007000100006.

16. Geib LTC. Determinantes sociais da saúde do idoso. Cien Saude Colet. 2012;17(1):123-33. http://dx.doi. org/10.1590/S1413-81232012000100015. PMid:22218546.

17. Fuchs VR. Social determinants of health: caveats and nuances. JAMA. 2017;317(1):25-6. http://dx.doi. org/10.1001/jama.2016.17335. PMid:28030707.

18. Piccini RX, Facchini LA, Tomasi E, Siqueira FV, Silveira DS, Thumé E, et al. Promotion, prevention and arterial hypertension care in Brazil. Rev Saude Publica. 2012;46(3):543-50. http://dx.doi.org/10.1590/ S0034-89102012005000027. PMid:22510974.

19. De Sá CAO, Corralo VS, Fachineto S, Schmidt CL, Cezar MA, Ribeiro CG. Obesidade, condição socioeconômica e hipertensão arterial no Extremo Oeste de Santa Catarina. Rev Salud Publica. 2014;16(2):184-94. http:// dx.doi.org/10.15446/rsap.v16n2.39536. PMid:25383493.

20. Brevidelli MM, Freitas FCG. Estudo ecológico sobre o desenvolvimento da saúde no Brasil. Cien Saude Colet. 2012;17(9):2471-80. http://dx.doi.org/10.1590/S1413-81232012000900027. PMid:22996897.

21. Garbois JA, Sodre F, Dalbello-Araújo M. Da noção de determinação social à de determinantes sociais da saúde. Saúde Debate. 2017;41(112):63-76. http://dx.doi.org/10.1590/0103-1104201711206.

22. Francisco PMSB, Segri NJ, Barros MBA, Malta DC. Desigualdades sociodemográficas nos fatores de risco e proteção para doenças crônicas não transmissíveis: inquérito telefônico em Campinas, São Paulo. Epidemiol Serv Saude. 2015;24(1):7-18. http://dx.doi.org/10.5123/S1679-49742015000100002.

23. Alves AC Jr. Consolidando a rede de atenção às condições crônicas: experiência da rede hiperdia de Minas Gerais. Inovação na gestão do SUS: experiências locais. Brasília (DF): Ministério da Saúde, Organização Pan-Americana de Saúde, Organização Mundial de Saúde; 2011.

24. Nobre ALCSD, Dias OV, Leite MTS, Freitas DM, Costa SM. Distributive justice in specialized health service and in access to medication. Rev Bioet. 2015;23(2):373-86. http://dx.doi.org/10.1590/1983-80422015232076.

25. Medeiros ARC, Araújo YB, Vianna RPT, Moraes RM. Modelo de suporte à decisão aplicado à identificação de indivíduos não aderentes ao tratamento anti-hipertensivo. Saúde Debate. 2014;38(100):104-18.

26. Brasil. Ministério da Saúde. Secretaria de Vigilância em Saúde. Departamento de Análise de Situação de Saúde. Plano de ações estratégicas para o enfrentamento das Doenças Crônicas Não Transmissíveis (DCNT) no Brasil 2011-2022. Brasília: Ministério da Saúde; 2011.

27. Pattussi MP, Hardy R, Sheiham A. The potential impact of neighborhood empowerment on dental caries among adolescents. Community Dent Oral Epidemiol. 2006;34(5):344-50. http://dx.doi.org/10.1111/j.16000528.2006.00283.x. PMid:16948673.

28. Comissão Nacional sobre Determinantes Sociais da Saúde. As causas sociais das iniquidades em saúde no Brasil: relatório final da Comissão Nacional sobre Determinantes Sociais da Saúde (CNDSS). Rio de Janeiro: Editora FIOCRUZ; 2008.

29. Andrade SSCA, Malta DC, Iser BM, Sampaio PC, Moura L. Prevalence of self-reported arterial hypertension in Brazilian capitals in 2011 and analysis of its trends in the period between 2006 and 2011. Rev Bras Epidemiol. 2014;17(Suppl 1):215-26. http://dx.doi.org/10.1590/1809-4503201400050017. PMid:25054265.

30. Pimenta HB, Caldeira AP. Fatores de risco cardiovascular do Escore de Framingham entre hipertensos assistidos por equipes de Saúde da Família. Cien Saude Colet. 2014;19(6):1731-9. http://dx.doi. org/10.1590/1413-81232014196.20092013. PMid:24897474.

31. Nieuwenhuijsen MJ, Khreis H, Triguero-Mas M, Gascon M, Dadvand P. Fifty shades of green: pathway to healthy urban living. Epidemiology. 2017;28(1):63-71. http://dx.doi.org/10.1097/EDE.0000000000000549. PMid:27525811.

32. Silveira IH, Junger WL. Green spaces and mortality due to cardiovascular diseases in the city of Rio de Janeiro. Rev Saude Publica. 2018;52:49. http://dx.doi.org/10.11606/S1518-8787.2018052000290. PMid:29723390. 